ON PLANNING AND FORECASTING THE LOCATION OF RETAIL AND SERVICE ACTIVITY

John R. Roy

CSIRO Division of Building Research, Melbourne, Australia

Börje Johansson

Regional and Urban Development Group, International Institute for Applied Systems Analysis, Austria

RR-85-6

May 1985

Reprinted from Regional Science and Urban Economics, volume 14 (1984)

INTERNATIONAL INSTITUTE FOR APPLIED SYSTEMS ANALYSIS

Laxenburg, Austria 
Research Reports, which record research conducted at IIASA, are independently reviewed before publication. However, the views and opinions they express are not necessarily those of the Institute or the National Member Organizations that support it.

Reprinted with permission from Regional Science and Urban Economics, 14(1984), 433-452.

Copyright @ 1984 Elsevier Science Publishers B.V. (North-Holland).

All rights reserved. No part of this publication may be reproduced or transmitted in any form or by any means, electronic or mechanical, including photocopy, recording, or any information storage or retrieval system, without permission in writing from the copyright holder.

Printed by Novographic, Vienna, Austria 


\section{FOREWORD}

The Regional Issues Project at the International Institute for Applied Systems Analysis (IIASA) contains a major study of metropolitan processes and policies. This report by John Roy and Börje Johansson presents models for analyzing formation of the infrastructure for retail and service activities in a metropolitan region, complementing another study by the same authors, together with Giorgio Leonardi, WP-84-47, published by IIASA. Roy and Johansson analyze the formation of infrastructure in the form of private facilities in a setting of noncooperative, oligopolistic competition between different types of economic agents. Thus, the report focuses on the policy formation of a regional planning authority in a so-called "mixed economy", in which priority is given to cooperative solutions of Pareto-type and to equity criteria with regard to the outcome between firms, landlords, and inhabitants of the region.

AKE E. ANDERSSON

Professor of Economics

Leader

Regional Issues Project 


\title{
ON PLANNING AND FORECASTING THE LOCATION OF RETAIL AND SERVICE ACTIVITY
}

\author{
John R. ROY* \\ CSIRO Division of Building Research, Melbourne, Australia \\ Börje JOHANSSON* \\ Regional and Urban Development Group, IIASA, Austria \\ Received October 1982, final version received January 1984
}

\begin{abstract}
In countries with mixed economies, planning authorities cannot usually enforce micro location and travel decisions of urban activities, but are confined to forming macro location and transport policy, within which agents can operate according to the market. An attempt is made here to describe such multilevel behaviour of the planning authority, landlords, firms and customers, and to obtain non-cooperative equilibria in terms of the controls exerted by each group. The behaviour of the market groups is described via entropy maximization, while alternative modes of behaviour are ascribed to the authority. In addition, a Pareto-type solution is examined.
\end{abstract}

\section{Introduction}

\subsection{A brief historical review}

In earlier work on shopping models, such as that of Lakshmanan and Hansen (1965), an iterative framework was suggested for determining shop locations by minimizing revenue/capacity disbalances. In his general treatise on entropy models, Wilson (1970) introduced shopping travel models, which were later generalized by Coelho and Wilson (1976), and Harris and Wilson (1978) to consider shop location simultaneously. Mathematical programming versions were presented by Macgill and Wilson (1979), and convergence and stability properties were investigated by Phiri (1980), Clarke (1981), Beaumont et al. (1981) and Rijk and Vorst (1983). Bertuglia and Leonardi (1980) described algorithms for handling log-accessibility transformations of the shopper objective under revenue/cost balance constraints. Recently, Leonardi $(1981 \mathrm{a}, \mathrm{b})$ presented a comprehensive approach to public facility location problems, in the context of his leadership of the Facility Location Task Force at IIASA (International Institute for Applied Systems Analysis).

\footnotetext{
* The authors are especially grateful to Paul Lesse, CSIRO Division of Building Research, Australia, for advice on the use of information theory concepts in such problems.
} 
In parallel to this development, disaggregated behavioural approaches to these problems have been proposed, such as by Blommestein et al. (1980). However, it is considered that these methods are currently best suited for use in impact analysis, rather than in the broader-brush physical planning problems where aggregate models have been used traditionally.

During the same period, considerable effort was being made in generalizing the form of entropy models, especially in considering the effects of a priori information and supply capacities. Contributions to this area were made, among others, by Fisk and Brown (1975), Dacey and Norcliffe (1976), Snickars and Weibull (1977), Batty (1978), Lesse et al. (1978), Brotchie et al. (1979) and Roy and Lesse (1981). Some of these ideas were applied to shopping models in Roy (1981).

\subsection{Motivations and scope of current paper}

There still remain significant differences between the model formulations suggested by major workers in the field. For instance, whereas Harris and Wilson (1978) define zonal attractiveness as the power $W_{j}^{\alpha}$ of endogenous floorspace terms $W_{j}$ for each centre $j$, eq. (39) of Leonardi (1981b) implies an attractiveness or exp $\left(-\lambda a_{j}\right)$, where $a_{j}$ is the exogenous operating cost per unit size in location $j$. In the current work, we account for zonal attractiveness in a way similar to Harris and Wilson (1978), but can also handle operating cost differentials in different zones within the planning authority objective. The model is derived using an entropy microstate approach, relating to distinct people making choices of distinct equivalent discrete amounts of shopping space, as already indicated in Roy (1981).

Most current model solutions, such as that in Harris and Wilson (1978), can be interpreted as finding equilibrium points of two player games consisting of customers making their shop choices according to entropy (or surplus) maximization, and a planning authority determining the location and size of new centres to ensure a proportionality between total turnover and floorspace, as described in Roy and Lesse (1983). Although such equilibrium models represented a considerable advance in the state of the art upon their inception, they still have deficiencies, especially their neglect of externality effects. In addition, by identifying the macro spatial policy of the planning authority with the micro location decisions of the retailers, not only is the freedom of the latter overconstrained, but one is unable to incorporate consistently both the (sub-optimal) profit maximizing behaviour of the retailers as well as efficiency and equity criteria for the planning authority. In the current work, we attempt to overcome these difficulties by interpreting the problem as finding non-cooperative Nash equilibria of three player games [Intriligator (1971)]. The behaviour of the shoppers is described essentially as in the works mentioned earlier, except that proxies for shopping comfort and 
convenience are introduced as 'qualitative' components of shopping centre attractiveness. The retailers are subdivided into various shop size clissses, and their locational behaviour is described by entropy maximization under constraints upon profits. The planning authority controls the overall location and size of new centres according to weighted sums of efficiency critcria, or via satisficing approaches producing compromises between efficiency and equity criteria in the sense of Nijkamp (1978). Alternatively, a positive model of the authority is introduced, in which entropy maximization is used to describe the authority's behaviour. Finally, the landlords (i.e., shopping centre developers) are not represented as a fourth player with an objective function. However, they appear in the game with a predetermined behaviour (strategy), influencing the results by either providing exogenous rents and unit infrastructure investment levels to the system, or by receiving viable rents (and corresponding investment levels) as output which reflect the expected profits to be made by the retailers. The flow and decision structure of the model is illustrated in fig. 1 .

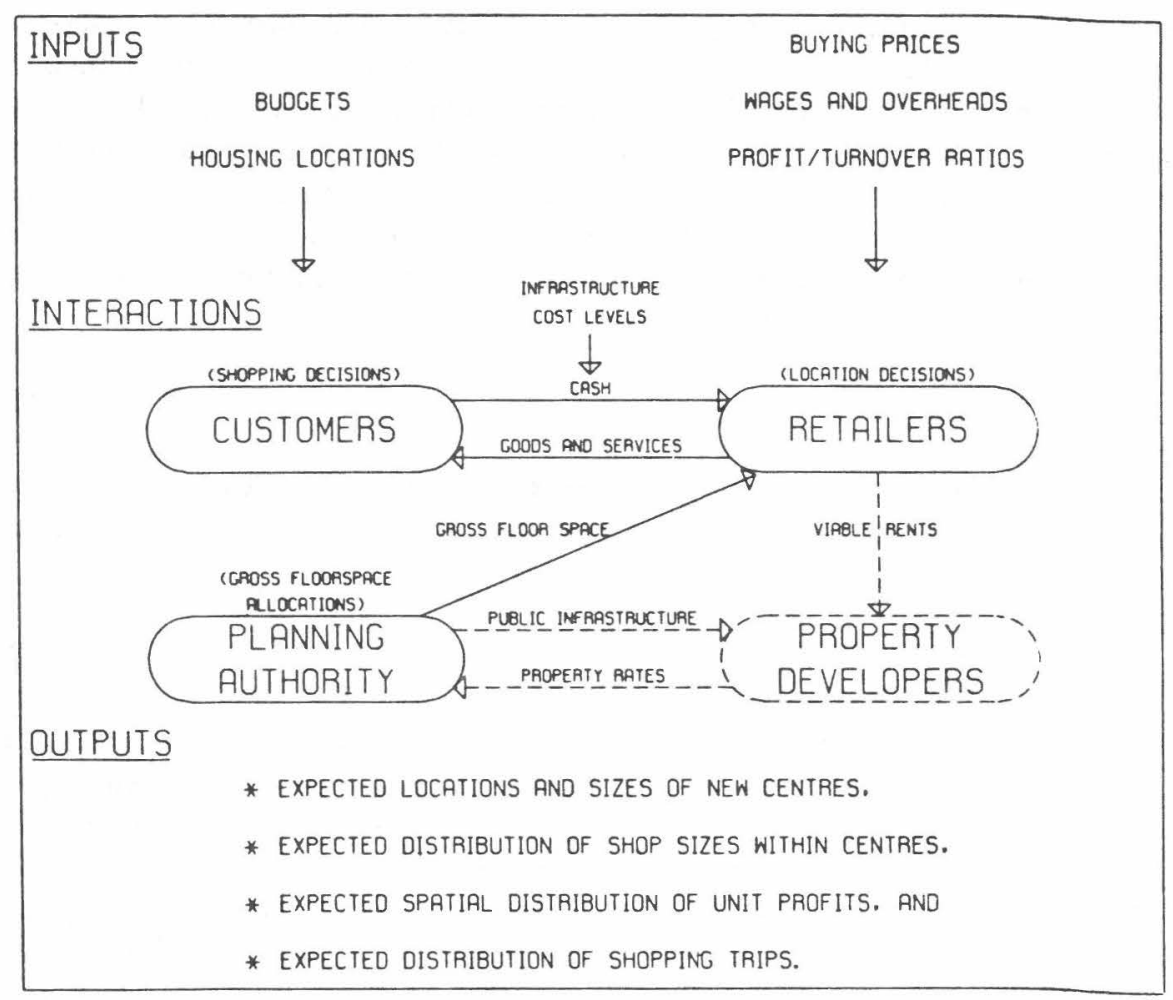

Fig. 1. Flow and decision structure of model. 
In addition to formulating the above equilibrium solutions, Pareto-type or cooperative solutions are also explored. These solutions represent the community's objective to coordinate the behaviour of all agents so as to reach a Pareto-satisfying result. Hence, pricing corrections to induce equilibrium need to be determined. In order to produce weighted objectives which include all three players, surplus representations for the players with entropy 'objectives' must be obtained. For entropy problems with more than one behavioural constraint, considerable judgement is required to select the most appropriate surplus form.

\section{Some basic relations}

\subsection{Shopper relations}

Consider that the potential shoppers are already located in residential zones $i$, and make $M_{i}$ shopping trips during a given time. In this work, we concentrate on shopping trips for basics, and assume that, on the average, a shopper habitually buys these basics at one of the available centres. In order to simplify the indexing, shoppers are not subdivided into socio-economic groups in this formulation. However, some spatial segregation of income groups can be incorporated by specifying an average budget $a_{i}$, taken to be available for shopping travel and shopping purchases for a typical resident in each zone $i$. Thus, if $c_{i j}$ are the average travel costs incurred between zone $i$ and centre $j$ and $d_{j}$ the parking charges (if any) at centre $j$, the average amount of money $y_{i j}$ available for purchases by a shopper from zone $i$ travelling to shop at centre $j$ is given as

$$
y_{i j}=a_{i}-c_{i j}-d_{i j} \quad \forall i, j .
$$

If average price levels $p_{j}$ for basics are given exogenously in each centre $j$ and $\bar{p}$ is the average price over all centres, the value $v_{i j}$ of goods purchased (normed to average price $\bar{p}$ ) is

$$
v_{i j}=\left(\bar{p} / p_{j}\right) y_{i j} \quad \forall i, j
$$

It is being assumed here that the price elasticity of demand is -1 , i.e., the amount of money spent by an individual on purchases in any centre $j$ is unaffected by the relative price levels $p_{j}$. However, the propensity to select a given centre $j$ is affected by its average price level.

In order to apply an entropy microstate approach to obtain a description of shopping travel, it is necessary to define a basic shop choice unit, characterized in Roy (1981) as an 'elemental' shop. In a sense, we seek a discrete representation, in terms of different shop 'size' classes $k$, of the usually non-linear relation between customer density and floorspace. From 


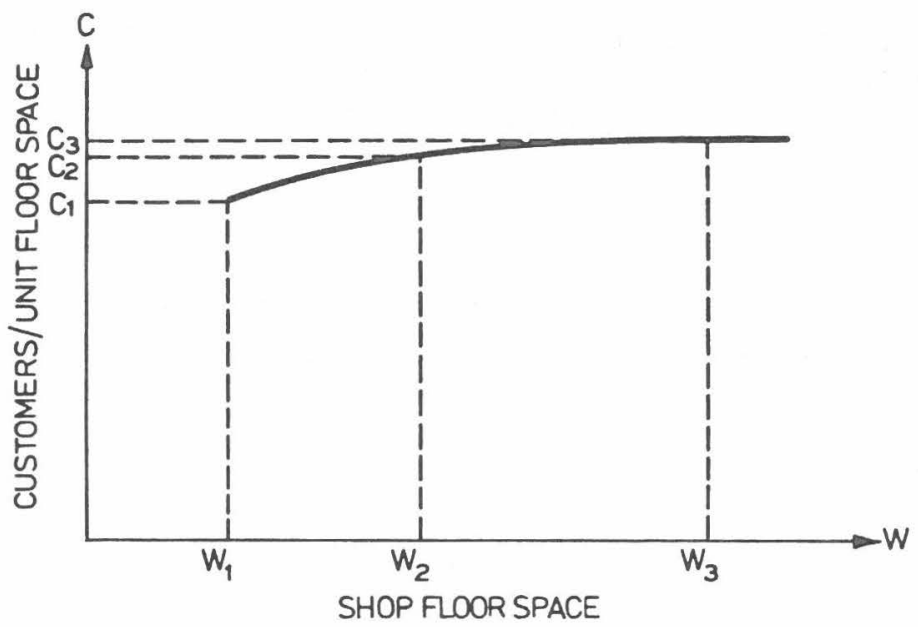

Fig. 2. Typical specifications for discrete shop size classes.

empirical data, a curve of expected customers/unit area is plotted against shop floorspace down to the average floorspace $w_{1}$ of the typical smallest shop where basics are purchased, as seen in fig. 2. Such a shop, with customers per unit area of $c_{1}$, is termed an elemental shop. Further shop size classes $k$ are defined to reasonably interpolate the continuous relation of fig. 2 , and the total number of new shops $F$ to be allocated are classified by size into groups $F_{k}$. In order to express the relative attractiveness power $n_{k}$ of a shop of size class $k$, we can see from fig. 2 that

$$
n_{k}=w_{k} c_{k} /\left(w_{1} c_{1}\right) \quad \forall k,
$$

where $n_{1}=1$ for an elemental shop. Note that, if the curve in fig. 2 were close to being a horizontal line $n_{k} \cong 1$ for all $k$, and only one shop class would be required.

\subsection{Retailer and landlord relations}

Consider the estimation of the number of shopping trips $S_{i j}$ between zone $i$ and centre $j$ as well as the number $F_{j k}$ of shops of size class $k$ choosing to locate in centre $j$ in addition to $F_{j k}^{0}$ shops already there. Due to varying accessibility to warehouses and bulk ordering arrangements, different averages buying prices $p_{j}^{*}$ may exist in different centres, and the transaction profits $w_{i j}$ from a typical customer from zone $i$ shopping in centre $j$ are given as

$$
w_{i j}=\left(1-p_{j}^{*} / p_{j}\right) y_{i j} \quad \forall i, j
$$


If $r_{j k}$ is the average rent per shop of class $k$ charged in centre $j$ and $g_{j k}$ the average wage plus overhead bill for a shop of class $k$ locating in $j$, then from eq. (1), the net profits $E_{j}$ accruing to centre $j$ become

$$
E_{j}=\sum_{i} S_{i j} w_{i j}-\sum_{k}\left(F_{j k}^{0}+F_{j k}\right)\left(r_{j k}+g_{j k}\right) \quad \forall j .
$$

Assuming that, on the average, profits within centres are distributed uniformly over their elemental shops, the average profit $e_{j k}$ per shop of class $k$ locating in centre $j$ is given as

$$
e_{j k}=n_{k} E_{j} / \bar{F}_{j},
$$

where $F_{j}$ is the number of elemental shops in centre $j$, that is,

$$
\bar{F}_{j}=\sum_{k} n_{k}\left(F_{j k}^{0}+F_{j k}\right) .
$$

If in any application, unit profits $e_{j k}$ in any centres $j$ are computed to fall below a certain 'shut-down level' $e_{k}{ }^{(-)}$for the retailers, then the exogenous rents $r_{j k}$ are too high, given $p_{j}$ and $g_{j k}$. From eq. (6), one may then determine a rent level such that $e_{j k} \geqq e_{k}^{(-)}$, enabling shops of type $k$ in such centres $j$ to become viable. Thereafter, the problem may be re-run with the reduced rent levels.

Also, if expected annual unit infrastructure investment levels $b_{j}$ per expected shopper are provided by the developers, the total new private investment $B$ comes out as

$$
B=\sum_{i j} b_{j} S_{i j}
$$

Rather than the rents $r_{j k}$ and wage plus overhead rates $g_{j k}$ being given exogenously by developers, an alternative approach is to let them be computed endogenously from the standpoint of the retailers. Then, if in certain centres $j$, these rents turn out to be lower than a value $r_{j k}{ }^{(-)}$required to provide a minimum viable return for the developers, the problem should be re-run using exogenous rents $r_{j k}{ }^{(-)}$for such centres via eq. (5). Letting the wage plus overheads costs be $\tau$ per expected customer, the assumptions in fig. 2 enable us to write $g_{j k}$ endogenously as

$$
g_{j k}=\tau n_{k}\left(\sum_{i} S_{i j}\right) / \bar{F}_{j} .
$$

Assume that the landlords charge (and are expected to charge) a certain proportion $\xi$ of the retailer's expected net unit profits (i.e., transaction profits 
minus wages and operating overheads) in rent. Then, given eq. (8), we obtain the following average endogenous rent level $r_{j k}$ in centre of

$$
r_{j k}=\xi_{n k}\left[\sum_{i} S_{i j} w_{i j}-\tau \sum_{i} S_{i j}\right] / \bar{F}_{j},
$$

which together with eq. (8), yields the profit relation (5) as

$$
E_{j}=(1-\xi)\left[\sum_{i} S_{i j}\left(w_{i j}-\tau\right)\right] \forall_{j}
$$

which, in contrast to eq. (5), just contains the shopper travel variables $S_{i j}$.

\section{Behaviour of main decision makers}

Except for the normative model of the planning authority, the behaviour of the key decision groups is described via entropy maximization. This provides a 'most probable' macro distribution in cases which may encompass diverse preferences, sub-optimal behaviour, heterogeneity across the options supplied and lack of perfect information. Note that, in contrast with most applications of game theory, it is not being assumed that such groups consciously maximize their entropy, but merely that entropy maximization provides a reliable description of their macro behaviour. After describing the statistics of the choice processes, constraint information expected to influence choice is introduced. The solution can be divided into two main phases, the estimating phase and the forecasting phase. In the estimating phase, the right-hand sides of these constraints are usually known, and Lagrange multipliers are determined which provide a 'best fit' to the observed behaviour. Then, these multipliers (analogously to elasticities) may be used in the forecasting phase, where the right-hand sides of the constraints emerge as outputs. Certain smoothness and regularity assumptions are required to justify such a procedure. In addition, for models which embed user behaviour within planning objectives, a reasonable continuity between present and future planning policy must apply. Occasionally, completely new policy information may become available for the forecasting situation, leading to new constraints with known right-hand sides being added at this latter stage. So long as such policy changes are not too severe, the estimated Lagrange multipliers on the existing constraints are probably still reliable. The behaviour of the three main players is now described.

\subsection{Customer behaviour}

Because of the heterogeneity of shoppers and price and other variations 
within shopping centres, shopper behaviour is usually more difficult to describe than that of the other agents. Firstly, both shoppers and their unit choices must be treated as distinct. Thus, from Roy (1981) and the definition of elemental shops in section 2.1, the number of potential choices $N_{T}$ by distinct shoppers each choosing a distinct elemental shop is given as the number of ways distinct shopper $M_{i}$ living in residential zones $i$ can choose among the

$$
\left[\sum_{k} n_{k}\left(F_{j k}^{0}+F_{j k}\right)\right]
$$

distinct elemental shops in shopping centres $j$ in groups $S_{i j}$, allowing more than one shopper per shop, yielding

$$
N_{T}=\left[\Pi_{i}\left(M_{i} ! / \Pi_{j} S_{i j} !\right)\right]\left[\Pi_{j}\left(\sum_{k} n_{k}\left(F_{j k}^{0}+F_{j k}\right)\right)^{\left(\sum_{i} s_{i j}\right)}\right] .
$$

Defining the entropy $S_{T}$ as $\log \left(N_{T}\right)$ and introducing the usual form of the Stirling approximation $\log x !=x(\log x-1)$, we obtain the following entropy maximization problem:

$$
\bar{S}_{T}=\max _{S_{i j}}-\sum_{i j} S_{i j} \log \left(S_{i j}\right) /\left[\sum_{k} n_{k}\left(F_{j k}^{0}+F_{j k}\right)\right]+\text { const., }
$$

under the origin balance constraint

$$
\sum_{j} S_{i j}=M_{i} \quad \forall i \quad \text { with the multipliers } \lambda_{i} .
$$

The search for other relevant constraints seem currently more of an art than a systematic procedure. A macro theory of choice (consumer behaviour) would be required to select the set of potentially most important constraints. Otherwise, the actual selection must rely on statistical criteria applied to empirical observations. The set of constraints which best succeeds in explaining observed behaviour should be adopted. The associated Lagrange multipliers constitute parameters of a behavioural model which may be used for forecasting applications. In this spirit, the following preliminary set of zontraints is chosen for the shop choice problem of eq. (12). Firstly, as we zonsider the value of goods which a shopper can obtain at any centre from his net shopping budget as a major factor in shopping centre choice, eq. (2) is used to write

$$
\sum_{i j} S_{i j} v_{i j}=V, \text { with multiplier } \alpha
$$


The effect of shopping travel time $t_{i j}$ between home and shops is included via the constraint

$$
\sum_{i j} S_{i j} t_{i j}=T, \text { with multiplier } v \text {. }
$$

As proxy for shopping 'convenience', we include information on the average time $m_{j}$ required to complete the shopping task in different centres $j$ using

$$
\sum_{i j} S_{i j} m_{j}=M, \text { with multiplier } \zeta .
$$

Finally, as a proxy for shopping 'comfort', the unit infrastructure investment levels $b_{j}$ in each centre are proposed, which are included via eq. (7) with multiplier $\kappa$.

\subsection{Retailer behaviour}

Retailers of different shop classes $k$ are free to locate in their preferred centres. If current or prior shop location probabilities $q_{j k}$ are available, then, following Snickars and Weibull (1977), retailer location behaviour may be described as finding the minimum information gain $\bar{I}$ according to the following process:

$$
\bar{I}=\min _{F_{j k}} \sum_{i j} F_{j k}\left[\log \left(F_{j k} / q_{j k}\right)\right]
$$

under the market clearing conditions

$$
\sum_{j} F_{j k}=F_{k} \quad \forall k \quad \text { with multipliers } \eta_{k} \text {, }
$$

where $F_{k}$ is the total number of class $k$ shops. Note that, if new shopping centres $j$ are being added to those existing when $q_{j k}$ was observed, the relevant probabilities for new centres should be given as

$$
\left(\sum_{l} q_{l k}\right) / N,
$$

where $N$ is the total number of centres, new plus existing. Then the original $q_{j k}$ are adjusted in proportion.

In addition, we impose demand-supply floorspace relations in the following form:

$$
\sum_{k} F_{j k} w_{k}=(1-r) W_{j} \text {, with multipliers } v_{j}
$$


where $r$ is the average shop vacancy rate and $W_{j}$ the amount of floorspace allocated by the authority to centre $j$. Eq. (19) links the macro location policy $W_{j}$ of the authority with the micro location decisions $F_{j k}$ of the retailers. If the vacancy rate $r$ were expected to be rather large, it may be preferable to endogenize zonal vacancy rates $r_{j}$ by incorporating the floorspace supply capacities $W_{j}$ directly into the objective of the retailers in eq. (17), as indicated in Roy (1981).

In addition to the above 'accounting' constraints, economic constraints need also be applied. For endogenous rents and wage plus overhead rates, the profit constraints of eq. (10) are applicable, which are attached to the retailer objective with estimated multipliers $\mu_{j}$. The mixed constraints of eq. (5) for exogenous rents may alternatively be applied. Note that, as mentioned earlier in section 2.2, the profit constraints may change between eq. (10) and (5), if the former produce non-viable rents for the landlords. In addition, exogenous rents $r_{j k}$ in eq. (5) may need to be reduced to ensure that certain retailers don't go bankrupt. If the landlords can't manage such reductions, the planning authority may well decide to intervene by the provision of corresponding rent subsidies.

\subsection{The planning authority}

It is considered that the planning authority has the power to allocate a total amount of new floorspace $W$ in amounts $W_{j}$ over shopping centres $j$.

Both a normative approach as well as a descriptive or positive approach can be adopted here. Within the normative procedure, either a weighted sum of efficiency criteria for the three groups is maximized, or strong equity criteria are introduced. Alternatively, a satisficing approach, as suggested by Nijkamp (1978), can be used to provide a compromise satisfaction of both equity and efficienty criteria. In the positive model, planning policy is described using an entropy or information gain objective. On the other hand, each outcome of the normative model is a policy suggestion (prescription). Note that, Leonardi (1981a, b) suggests the use of a consumer surplus minus operating cost objective for the planning authority. However, we believe that the consumer surplus objective is adequately catered for by the role of the customers in the game, and that the authority's policy can here be directed towards satisfying overall efficiency and welfare criteria which cannot be guaranteed by the market. For our positive model, the entropy objective of the authority can be regarded as a type of producer surplus.

In our general formulation of the normative procedure, an efficiency criterion referring to all three groups is given as a weighted sum $Z$ of transaction profits minus shopping travel costs minus operating plus annual capital costs $z_{j}$ of the required public infrastructure. This rather altruistic policy of the planning authority presumes that it is constrained in some way to act in the 'public interest'. The objective is as follows: 


$$
\bar{Z}=\max _{w_{j}}\left\{\left[\sum_{i j} S_{i j}\left(\alpha_{1} w_{i j}-\alpha_{2} c_{i j}\right)\right]-\left(1-\alpha_{1}-\alpha_{2}\right) \sum_{j} W_{j} z_{j}\right\},
$$

where $\alpha_{1} \geqq 0, \alpha_{2} \geqq 0$ and $\left(\alpha_{1}+\alpha_{2}\right) \leqq 1$. The weightings reflect importance judgements for the three criteria as well as their relative expected total contributions to the objective. If any floorspace allocations $W_{j}$ fall below a minimum viable level $W_{\min }$, such potential centres should be omitted and the analysis repeated.

As a market clearing constraint is associated with eq. (20), a maximum of $(N-2)$ extra independent constraints can be applied before the system becomes over-determined in terms of its $N$ control variables $W_{j}$. For instance, if equity criteria of equal units profits $e$ per elemental shop for each centre are to be imposed, this introduces $(N-1)$ independent relations and renders eq. (20) superfluous. From eq. (5), equalizing the profits $e$ per elemental shop would yield the conditions

$$
\sum_{i} S_{i j} w_{i j}=\left[\sum_{k}\left(F_{j k}^{0}+F_{j k}\right)\left(r_{j k}+g_{j k}+n_{k} e\right)\right] \quad \forall_{j},
$$

from which the unknown $e$ value can be eliminated by summing over $j$. On the other hand, equity in average value of goods purchased per unit total zonal budget for each residential zone $i$ implies the following conditions

$$
\sum_{j} S_{i j} v_{i j}=v M_{i} a_{i} \quad \forall_{i}
$$

where $v$ is the unknown value ratio of goods purchased per unit total budget for a resident in any zone, eliminated by summing over $i$. Alternatively, we may try to equalize the average shopping travel time per elemental shop for each zone $i$. However, if the number of residential zones $M$ is greater than the number of shopping centres $N$, the authority has insufficient policy instruments $W_{j}$ to fulfil either of these criteria perfectly.

For a satisficing approach, the objective of eq. (20) is used, as well as requiring that the relative spread between maximum and minimum values of unit profits $e_{j}$ or relative zonal purchased goods value levels $v_{i}$ should not exceed certain rations $\delta_{1}$ and $\delta_{2}$ respectively. So long as $\delta_{1}$ and $\delta_{2}$ are not made too small, less than $(N-2)$ of the relevant constraints

$$
e_{j} \leqq\left(1+\delta_{1}\right) e_{j}^{\text {min }} \quad \forall_{j}
$$

as well as

$$
v_{i} \leqq\left(1+\delta_{2}\right) v_{i}^{\min } \quad \forall_{i}
$$

R.S.U.E. - F 
will be active, and system (20), so constrained, will not be overdetermined. Note that, an iterative approach must be used here, as successive guesses need to be made of the worst centres and zones and their respective unit profit and goods value levels by running system (20) initially as unconstrained.

Finally, the case of a so-called 'positive' model of the authority is examined, where no explicit objective or set of objectives has been conveyed to the planner, but he must infer authority policy when making forecasts.

Assuming a prolongation of past policy decisions, the analyst may use a Kullback information gain objective to describe the behaviour of the authority, using the approach suggested by Snickars and Weibull (1977). Thus, if the observed or a priori spatial policy probabilities $q_{j}$ are defined as $\left(W_{j}^{0} / W^{0}\right)$, the 'objective' $\bar{I}$ for the authority is to find the new planning policy $W_{j}$ which implies minimum divergence from its past policy, in the form

$$
\bar{I}=\min _{W_{j}} \sum_{j} W_{j} \log \left(W_{j} / q_{j}\right) .
$$

If new centres are to be created, then equal probabilities $q_{j}=(1 / N)$ are assigned for these centres and the $q_{j}$ 's for existing centres adjusted in proportion, such that the relation $\sum_{j} q_{j}=1$ is maintained. This expression replaces eq. (20) of the normative model.

Direct use of eq. (25) for the positive model will imply a projection of observed planning policy into the future. However, if it is expected that any parameters in the system which will directly affect planning decisions are to change significantly in relative terms during the forecast period, constraints containing such parameters should be attached to eq. (25), and their multipliers obtained in the estimating phase.

\section{Some interactive equilibrium solutions}

\subsection{Efficiency objective for the planning authority}

If the authority is taken to have the efficiency objective of eq. (20), the first solution step is to differentiate partially the entropy objective, eq. (12), of the 'lowest level' player, i.e., the customers, with respect to their decision variables $S_{i j}$, including constraints (7) and (13) to (16). This process yields for $S_{i j}$

$$
S_{i j}=\bar{F}_{j} e^{-\lambda_{i}} e^{-\phi_{i j}},
$$

where, in forecasting applications, $\phi_{i j}$ is the known sum $\left(\alpha v_{i j}+v t_{i j}+\zeta m_{j}+\kappa b_{j}\right)$. Now, eq. (17) can be differentiated with respect to the retailer decision 
variables $F_{j k}$, together with constraints (18) and (19). However the profit constraints (5) or (10) must be applied also, whereby eq. (26) is used to make them functions of $F_{j k}$ alone. Considering firstly the case of endogenous rents in eq. (19), and eliminating $\lambda_{i}$ from eq. (26) via constraints (13), we finally obtain

$$
F_{j k}=q_{j k} h_{j}^{n_{k}} B_{j}^{w_{k}} C_{k},
$$

where

$$
\begin{aligned}
& B_{j}=e^{-v_{j}}, \quad e^{-\eta_{k}} \quad \text { and } \\
& \quad \ln h_{j}=-\left\{\sum_{i}\left[u_{i j} /\left(\sum_{j} \bar{F}_{j} r_{i j}\right)\right]-\left[r_{i j}\left(\sum_{j} \bar{F}_{j} u_{i j}\right) /\left(\sum_{j} \bar{F}_{j} r_{i j}\right)^{2}\right]\right\}, \\
& \quad \text { with } r_{i j}=e^{-\phi_{i j}} \text { and } u_{i j}=M_{i} \mu_{j} r_{i j}(1-\xi)\left(w_{i j}-\tau\right) .
\end{aligned}
$$

From eq. (19) and (27), $W_{j}$ is seen to be expressible monotonically in terms of $B_{j}$ and $C_{k}$, which with $A_{i}=e^{-\lambda_{i}}$, allows the planning objective (20) to be given as

$$
\begin{aligned}
\bar{Z}= & \max _{A_{i}, B_{j}, c_{k}}\left\{\left[\sum_{i j}\left(\alpha_{1} w_{i j}-\alpha_{2} c_{i j}\right) A_{i} r_{i j}\left(\sum_{k} n_{k}\left(F_{j k}^{0}+q_{j k} h_{j}^{n_{k}} B_{j}^{w_{k}} C_{k}\right)\right)\right] .\right. \\
& \left.-\left(1-\alpha_{1}-\alpha_{2}\right) \sum_{j} z_{j}\left(\sum_{k} w_{k} h_{j}^{n_{k}} q_{j k} B_{j}^{w_{k}} C_{k}\right) /(1-r)\right\}
\end{aligned}
$$

with eq. (18) formed

$$
\sum_{j} q_{j k} h_{j}^{n_{k}} B_{j}^{w_{k}} C_{k}=F_{k} \quad \forall_{k},
$$

eq. (19) in the form

$$
\sum_{j k} q_{j k} h_{j}^{n_{k}} w_{k} B_{j}^{w_{k}} C_{k}=(1-r) W,
$$

and eq. (13), using relations (26) and (27), as

$$
\sum_{j} A_{i} r_{1 j}\left[\sum_{k} n_{k}\left(F_{j k}^{0}+q_{j k} h_{j}^{n_{k}} B_{j}^{w_{k}} C_{k}\right)\right]=M_{i} \quad \forall i .
$$

The above system and its constraints may be solved as an iterative sequence of geometric programs [Beightler and Phillips (1976)], where the value of the $h_{j}$ term $^{\prime}$ is re-evaluated after every iteration - it is still necessary to 
demonstrate convergence for this process. Alternatively, writing eq. (27) as $f\left(F_{j k}, B_{j}, C_{k}\right)=0$, we may use the calculus to write $\partial F_{j k} / \partial B_{j}=$ $-\left(\partial f / \partial B_{j}\right) /\left(\partial f / \partial F_{j k}\right)$ and $\partial F_{j k} / \partial C_{k}=-\left(\partial f / \partial C_{k}\right) /\left(\partial f / \partial F_{j k}\right)$ and solve the system by a linearized gradient approach. Numerical experiments will need to be carried out to see if the above procedures (or others) are reliable and efficient. After final solution for the $A_{i}, B_{j}$, and $C_{k}$ variables, the expected retailer location decisions $F_{j k}$ may be obtained directly from eq. (27), the corresponding macro planning policy variables $W_{j}$ from eq. (19) and the expected shopping decisions $S_{i j}$ from eq. (26). In addition, other information such as unit rent and staffing levels, unit profit levels, expected total infrastructure costs and zonal accessibility values may be readily computed.

Note that, as the structure of this problem has some analogies with that of the two player game implied in the work of Harris and Wilson (1978), positive interior equilibrium solutions may not necessarily exist, especially when the curve in fig. 2 has a strong upward gradient. However, solutions with some of the $W_{j}$ values equal to zero may still exist, and may be useful if it is not required a priori to establish centres at all potential locations $j$.

For the case of exogenous rents in eq. (5), the recommended solution procedure would be the same as for endogenous rents above. The only change would be the structure of the term $h_{j}$.

\subsection{A positive model of the planning authority}

In this case, except for the inclusion of any extra constraints containing policy parameters which are expected to change significantly over time, ${ }^{1}$ observed planning policy is essentially projected into the future. Using eq. (19) and (27), eq. (25) may be transformed to

$$
\begin{aligned}
\bar{I}= & \min _{B_{j}}, c_{k}\left\{\sum_{j}\left[\sum_{k}\left(w_{k} q_{j k} h_{j}^{n_{k}} B_{j}^{w_{k}} C_{k}\right) /(1-r)\right]\right. \\
& \left.\times\left[-\log q_{j}+\log \left(\sum_{k}\left(\mathrm{w}_{k} q_{j k} \mathrm{~h}_{j}^{n_{k}} B_{j}^{w_{k}} C_{k}\right) /(1-r)\right)\right]\right\}
\end{aligned}
$$

under the constraints (29) and (30). Constraints (31) would not be required, as the expression (25) does not contain the shopper travel variables $S_{i j}$ explicitly. The multiplier $\lambda_{i}$ should be eliminated already from eq. (32) and its constraints no longer represent a conventional geometric program, thus either requiring the design of a specific algorithm for this problem or the use of a gradient approach.

\footnotetext{
${ }^{1}$ Such a constraint may be an operating budget limitation $\sum_{j} W_{j} z_{j}=\bar{Z}$. Inclusion of this would overcome the objection of Leonardi (1981b, bottom of p. 1095), as it would encourage concentration in low cost centres for low operating budgets $\boldsymbol{Z}$.
} 
The resulting solutions for $W_{j}, F_{j k}$ and $S_{i j}$ would then represent the expected behaviour of the system if the planning authority continued with its observed past policy. Any inequities or possible instabilities of the forecast solution could be explained to the authority, which may then decide on certain satisficing constraints etc. in an attempt to counteract these tendencies.

\subsection{Models to enhance equity properties of the system}

If the location policy $W_{j}$ is used to ensure equal average unit net profits $e$ in each centre, the retailers (if they are provided with this information) will ostensibly have no average incentive to move in the forseeable future, and a competitive equilibrium would be sustained. Eliminating $e$ from eq. (21) by summing over $j$, eq. (5) or (10) may be used to write

$$
\left(\sum_{j} E_{j}\right) \bar{F}_{j} / \bar{F}-E_{j}=0 \quad \forall_{j},
$$

where $\bar{F}$ is the known total number of elemental shops to be allocated. As the policy variables $W_{j}$ are 'replaced' by the conditions of eq. (33), constraint (19) is no longer required in the retailer objective of eq. (17). Constraints (33) replace constraints (5) or (10), but the multipliers are unknown here, as the equity conditions are a planning prescription. This contrasts with the previous two sections, where the multipliers $\mu_{j}$ on constraints (5) or (10) were estimated according to observed profit levels $E_{j}$, and then used directly in the forecasting phase. A Newton-Raphson procedure may be used to solve this problem for $F_{j k}$, after which the policy variables $W_{j}$ can bc obtained directly from eq. (19). Convergence properties of the procedure can be tested empirically.

As explained in the consideration of constraints (23) and (24), a satisficing approach may be adopted to obtain a compromise between equity measures on profits and value ratios of purchased goods and the efficiency objective of eq. (20). Such constraints would replace the profit constraints of eq. (5) or (10). However, the satisficing solution is probably numerically the most complicated, and much research will be required to obtain efficient and reliable algorithms. This is a worthwhile goal, as the ability to reasonably satisfy several criteria simultaneously seems most desirable.

\subsection{A market type model}

In some cases, the planning authority may not possess the power to allocate quantities of floorspace to new or existing centres, but may merely be able to designate certain spots throughout the urban area as potential 
sites for such centres. In that case, the problem would essentially reduce to a two-player game between the customers and retailers. The customer objective would be given from eq. (26), and in the absence of constraint (19), the implicit retailer result would correspond to eq. (27) with the $\boldsymbol{B}_{j}$ term absent. One possible solution method may be to make an estimate of the $F_{j k}$ distribution, compute the factors $h_{j}$, re-compute the $F_{j k}$ válues, and so on, until convergence is obtained. Otherwise a Newton-Raphson procedure may have to be used. Then, the customer solution for $S_{i j}$ can be obtained from eq. (26) using constraint (13). Finally, the expected floorspace requirements could be evaluated from eq. (19).

Such a market solution would be of particular value to individual retailers and developers, as it could evaluate impact in cases where several competitiors simultaneously were planning construction of new centres, and one was uncertain of their expected location decisions in respect of alternative locations of one's own planned centre or centres. Of course, the solution may yield non-viable profit levels in certain centres, implying instability in the longer term.

\section{Some cooperative solutions}

\subsection{Problem formulation}

In this section, a quasi-cooperative type of model is presented. From this model, one may derive policy measures which the community may use to coordinate the behaviour of all three agents, in such a way that a cooperative solution is obtained. The model may be used to generate a set of scenarios, each satisfying the Pareto criterion. It is then possible to compare the solutions and the associated policy measures.

The model may be summarized as follows. A weighted sum of the objectives of the three players, i.e., the shoppers, retailers and planning authority, is maximizing simultaneously in terms of the decision variables of each player. As the efficiency objective for the planning authority, eq. (20), is expressed in monetary units, the entropy 'objectives' of the shoppers and retailers must also be transformed into an equivalent monetary form. Such equivalent surplus measures have already been derived for entropy problems with one cost constraint (usually a travel cost constraint), as illustrated by Champernowne, Williams and Coelho (1976). For instance, when the righthand side of the constraint is $\bar{C}$ and the estimated Lagrange multiplier $\beta$, the surplus is given as $(S(\beta-\bar{C})$, where $S$ is the system entropy. In our case with multiple constraints, it was decided to use the constraint (14) on the net value of shopping purchases for the shoppers, as this is the most important item for the customer. Thus, from eqs. (12), (26) and (14) to (16), the shopper surplus $U_{T}$ is given as 


$$
\begin{aligned}
U_{T}= & -(1 / \alpha)\left\{\sum_{i j} S_{i j} \log \left(S_{i j} / \bar{F}_{j}\right)\right\}-\sum_{i j} S_{i j} v_{i j} \\
& -(1 / \alpha)\left[\sum_{i j} S_{i j}\left(v t_{i j}+\zeta m_{j}+\kappa b_{j}\right)\right] .
\end{aligned}
$$

On the other hand, as profit maximization can be considered such a predominant motivation for the retailers, we choose to define the retailer benefit $U_{L}$ from eq. (10) directly as the total profit

$$
U_{L}=\sum_{i j} S_{i j}\left(w_{i j}-\tau\right)(1-\xi)
$$

The criterion of the planning authority is taken as the combined efficiency objective $Z$ of eq. (20), which indicates the authority's natural desire to minimize its own system operating costs, as well as a degree of 'benign interest' in the welfare of the participating firms and customers. ${ }^{2}$ Letting $\omega_{1}$ be the community's exogenous weighting on shopper utility and $\omega_{2}$ that on retailer profits, the cooperative or Pareto-type problem may be defined as finding the maximum total community utility $\bar{U}$, in the form

$$
\bar{U}=\max _{S_{i j}, F_{j k}, w_{j}}\left[\omega_{1} U_{T}+\omega_{2} U_{L}+\left(1-\omega_{1}-\omega_{2}\right) Z\right]
$$

under constraints (13), (18) and (19). The relation $\partial U / \partial S_{i j}=0$ with constraints (13) yields a similar expression to eq. (26), except that index $\phi_{i j}$ is replaced by $\psi_{i j}=\left(\phi_{i j}-\alpha / \omega_{1}\left[\omega_{2}\left(w_{i j}-\tau\right)(1-\xi)+\left(1-\omega_{1}-\omega_{2}\right)\left(\alpha_{1} w_{i j}+\alpha_{2} c_{i j}\right)\right]\right)$. Also $\partial U / \partial F_{j k}=0$ with constraints (18) and (19) yields

$$
\sum_{i} S_{i j} / \bar{F}_{j}=\left(\eta_{k}+w_{k} v_{j}\right) / n_{k}=k_{j} \quad \forall_{j}
$$

which implies, in this case of endogenous rents, that the same relative mix of shops of each class $k$ may occur in each centre $j$, that is

$$
F_{j k}=\left(\sum_{k} n_{k} F_{j k}\right) F_{k} /\left(\sum_{k} n_{k} F_{k}\right) \quad \forall j, k .
$$

Assuming that $W_{j}>0$ for all $j, \partial U / \partial W_{j}=0$ with constraint (19) yields $v_{j}=$ $-\left(1-\omega_{1}-\omega_{2}\right)\left(1-\alpha_{1}-\alpha_{2}\right) z_{j} /(1-r)$. With this relation and eqs. (18) and (38), the ratios $\sum_{i} S_{i j} / \bar{F}_{j}$ may be evaluated in eq. (37), which may then be solved

\footnotetext{
${ }^{2}$ As the community is acting as the coordinator of the Pareto policy, it may be sometimes more realistic to give here the authority's objective $Z$ as just the maximization of its own operating surplus.
} 
with the modified eq. (26) simultaneously for $S_{i j}$ and $\bar{F}_{j}$, using the matrix approach presented by Harris and Wilson (1978). Thereafter, eq. (38) yields the retailer locations $F_{j k}$ and eq. (19) the macro planning policy variables $W_{j}$.

\subsection{Implementation policy}

Although the new macro retail location policy $W_{j}$ could be imposed on the planning authority, the cooperative solutions for shop choice $S_{i j}$ and retailer location decisions $F_{j k}$ will not be in equilibrium, either with each other or in terms of the new macro location policy. For the $S_{i j}$ case, this is due to the modification of the $\phi_{i j}$ terms in eq. (26) to $\psi_{i j}$. For $F_{j k}$, the solution of eq. (27) is obviously different to that of the cooperative case above for given $S_{i j}$ and $W_{j}$.

In order to bring the cooperative solutions for $S_{i j}$ and $F_{j k}$ into equilibrium, certain pricing corrections are required. For $S_{i j}$, one approach may be to use road or route pricing corrections $\Delta c_{i j}$ on the shopping travel costs $c_{i j}$, such that the market response term $\phi_{i j}$ of eq. (26) for modified prices $\left(c_{i j}\right.$ $+\Delta c_{i j}$ ) equals the required Pareto response term $\psi_{i j}$ (see above) for the original prices $c_{i j}$. These relations enable us to solve for the corrections $\Delta c_{i j}$. Road or route pricing may be extremely difficult to implement, even for public transport systems. An alternative is to approximate the effect by applying parking fee corrections $\Delta d_{j}$ of $\left(\sum_{i} S_{i j} \Delta c_{i j}\right) /\left(\sum_{i} S_{i j}\right)$. On the other hand, the retailer location solution may be equilibrated by introducing rent corrections $\Delta r_{j k}$ using the sensitivities $\mu_{j}$ on the profit constraints.

It is well known that the cooperative (Pareto) solution enables a greater sum of benefits $U$ to be achieved than the non-cooperative case. Nevertheless, a trade-off would need to be made between these expected extra benefits and the actual costs to the community of administering the differential coordinating pricing policies for parking fees and shop rents.

\section{Conclusions and recommendations}

A model framework has been presented which integrates the objectives of customers, firms and the planning authority in the location of new shopping and service centres. Certain one-way interactions with the landlords or property developers are included also. Within this framework, both a satisficing approach as well as a positive model may be selected with regard to the planning authority. In essence, the system consists of a set of coupled entropy models, where most of the Lagrange multipliers on cost or behavioural constraints are already obtained during the estimation phase, but the multipliers on the accounting or balance constraints are evaluated during the solution phase for the 3-player game. However, if reliable independent forecasts exist for the right-hand sides of any behavioural or 
policy constraints (e.g., budgets and resource consumption values), these should be applied during the solution phase. The micro decisions of customers, firms and landlords are embedded within macro spatial policy decisions of the planning authority. Although, as such, the models represent an advance on those currently available, several problems need further attention.

An immediate priority is to develop efficient estimation procedures which can handle multiple economic constraints, such as eq. (7) and (14) to (16) for $S_{i j}$. Although some of the new models can be solved by known techniques, such as geometric programming and matrix approaches [see Harris and Wilson (1978), and Phiri (1980)], others require special iterative procedures, for which convergence needs to be demonstrated. Also certain nonuniqueness problems and exterior solutions need to be examined [Rijk and Vorst (1983)].

Regarding the structure of the models, attempts should be made to include the landlords as a fourth set of actors in regard to their rent and investment decisions. For the retailers, one may consider endogenizing the average price levels $p_{j}$ in each centre. In addition, for situations where there is a rapid turnover of retailers within centres, the strong market clearing assumptions of eq. (19) can be relaxed, and the vacancy rates $r_{j}$ treated as endogenous.

Finally, in the longer term, shopping travel should be handled within the framework of activity analysis. In addition, qualitative aspects should be allowed to affect retailer and customer decisions by using multi-dimensional scaling techniques at the aggregate level. Above all, more time series data should be obtained, such that certain key assumptions may be validated. A study of the stability over time of the Lagrange multipliers is one such issue, which is also connected with the theory of choice and general systems theory.

\section{References}

Batty, M., 1978, Speculations on an information theoretical approach to spatial representation in: I. Masser and P. Brown, eds., Studies in applied regional science, Vol. 10, Spatial representation and spatial interaction (Martinus Nijhoff, Leiden) 115-147.

Beaumont, J.R., M. Clarke and A.G. Wilson, 1981, Changing energy parameters and the evolution of urban spatial structure, Regional Science and Urban Economics 11, 287-315.

Beightler, C.S. and D.T. Phillips, 1976, Applied geometric programming (Wiley, New York).

Bertuglia, C.S. and G. Leonardi, 1980, Heuristic algorithms for the normative location of retail activities systems, Papers of the Regional Science Association 44, 149-159.

Blommestein, H., P. Nijkamp and E. van Veenendaal, 1980, Shopping perceptions and preferences: A multidimensional attractiveness analysis of consumer and entrepreneurial attitudes, Economic Geography 56, 155-174.

Brotchie, J.F., P.F. Lesse and J.R. Roy, 1979, Physics, economics and planning - A new generation of urban and building models, in: PArC 79, Proceedings of the int. conference on applications of computers in architecture, building design and urban planning (AMK Berlin) 505-516.

Champernowne, A.F, H.C.W.L. Williams and J.D. Coelho, 1976, Some comments on urban travel demand analysis, model calibration and the economic evaluation of transport plans, Journal of Transport Economies and Policy 10, 267-285.

RSUE-G 
Clarke, M., 1981, A note on the stability of equilibrium solutions of production-constrained spatial-interaction models, Environment and Planning A 13, 601-604.

Coelho, J.D. and A.G. Wilson, 1976, The optimum location and size of shopping centres, Regional Studies 10, 413-421.

Dacey, M.P. and A. Norcliffe, 1976, New entropy models in the social sciences, 1: Elementary residential-location models, Environment and Planning A 8, 299-310.

Fisk, C. and G.R. Brown, 1975, A note on the entropy formulation of distribution models, Operational Research Quarterly 26, 755-758.

Harris, B. and A.G. Wilson, 1978, Equilibrium values and dynamics of attractiveness terms in production-constrained spatial interaction models, Enviroment and Planning A 10, 371-388.

Intriligator, M.D., 1971, Mathematical optimization and economic theory (Prentice-Hall, Englewood Cliffs, NJ).

Lakshmanan, T.R. and W.G. Hansen, 1965, A retail market potential model, Journal of the American Institute of Planners 31, 134-143.

Leonardi, G., 1981a, A unifying framework of public facility location problems - part 1: A critical overview and some unsolved problems, Environment and Planning A 13, 1001-1028.

Leonardi, G., 1981b, A unifying framework for public facility location problems - part 2: Some new models and extensions, Environment and Planning A 13, 1085-1108.

Lesse, P.F., J.F. Brotchie, J.R. Roy and R. Sharpe, 1978, A new philosophy for regional modelling, Papers of the third meeting of the Australian and New Zealand Section, Regional Science Association (Australian and New Zealand Section, Regional Science Association, Melbourne) 165-178.

Macgill, S.M. and A.G. Wilson, 1979, Equivalences and similarities between some alternative urban and regional models, Sistemi Urbani 1, 9-40.

Nijkamp, P., 1978, Compromise choices in spatial interaction and regional planning models, in: Karlqvist et al., eds., Spatial interaction theory and planning models (North-Holland, Amsterdam).

Phiri, P.A., 1980, Calculation of the equilibrium configuration of shopping facility sizes, Environment and Planning A 12, 983-1000.

Rijk, F.A. and A.C.F. Vorst, 1983, On the uniqueness and existence of equilibrium points in an urban retail model, Environment and Planning A 15, 475482.

Roy, J.R., 1981, A note on consistency of choice units in entropy models, Transportation Research B 15, 159-164.

Roy, J.R. and P.F. Lesse, 1981, On appropriate microstate descriptions in entropy modelling, Transportation Research B 15, 85-96.

Roy, J.R. and P.F. Lesse, 1983, Planning models for non-cooperative solutions: A two-player game approach, Regional Science and Urban Economics 13, 195-211.

Snickars, F. and J.W. Weibull, 1977, A minimum information principle: Theory and practice, Regional Science and Urban Economics 7, 137-168.

Wilson, A.G., 1970, Entropy in urban and regional modelling (Pion, London). 